\title{
Validating the WHO Maternal Near Miss Tool in a high-income country
}

\author{
TOM WITTEVEEN ${ }^{1}$, ILONA DE KONING ${ }^{1}$, HANS BEZSTAROSTI ${ }^{1}$, THOMAS VAN DEN AKKER ${ }^{1}$, \\ JOS VAN ROOSMALEN ${ }^{1,2} \&$ KITTY W. BLOEMENKAMP ${ }^{1}$ \\ ${ }^{1}$ Department of Obstetrics, Leiden University Medical Center, Leiden, and ${ }^{2}$ Athena Institute, VU University Amsterdam, \\ Amsterdam, the Netherlands
}

\begin{abstract}
Key words
Severe acute maternal morbidity, maternal health, Maternal Near Miss Tool, World

Health Organization, high income country, organ dysfunction

\section{Correspondence \\ Tom Witteveen, Department of Obstetrics, K- 6-P-35, Leiden University Medical Centre, \\ P.O. Box 9600, 2300 RC Leiden, the \\ Netherlands. \\ E-mail: tom.witteveen@gmail.com}

\section{Conflict of interest}

The authors have stated explicitly that there are no conflicts of interest in connection with this article.

Please cite this article as: Witteveen $T$, de Koning I, Bezstarosti $\mathrm{H}$, van den Akker $\mathrm{T}$, van Roosmalen J, Bloemenkamp KW. Validating the WHO Maternal Near Miss Tool in a highincome country. Acta Obstet Gynecol Scand 2016; 95:106-111.
\end{abstract}

Received: 15 August 2015

Accepted: 19 September 2015

DOI: 10.1111/aogs.12793

\begin{abstract}
Introduction. This study was performed to assess the applicability of the WHO Maternal Near Miss Tool (MNM Tool) and the organ dysfunction criteria in a high-income country. Material and methods. The MNM tool was applied to 2552 women who died of pregnancy-related causes or sustained severe acute maternal morbidity between August 2004 and August 2006 in one of the 98 hospitals with a maternity unit in the Netherlands. Fourteen $(0.6 \%)$ cases had insufficient data for application. Each case was assessed according to the three main "MNM categories" specified in the MNM tool and their subcategory criteria: five disease-, four intervention- and seven organ dysfunction-based criteria. Potentially life-threatening conditions (disease-based inclusions) and lifethreatening cases (organ dysfunction-based inclusions) were differentiated according to WHO methodology. Outcomes were incidence of all (sub)categories and case-fatality rates. Results. Of the 2538 cases, 2308 (90.9\%) women fulfilled disease-based, 2116 (83.4\%) intervention-based and $1024(40.3 \%)$ organ dysfunction-based criteria. Maternal death occurred in 48 women, of whom 23 (47.9\%) fulfilled disease-based, 33 (68.8\%) intervention-based and 31 $(64.6 \%)$ organ dysfunction-based criteria. Case-fatality rates were 23/2308 $(1.0 \%)$ for cases fulfilling the disease-based criteria, 33/2116 (1.6\%) for intervention-based criteria and 31/1024 (3.0\%) for women fulfilling the organ dysfunction-based criteria. Conclusions. In the Netherlands, where advanced laboratory and clinical monitoring are available, organ dysfunction-based criteria of the MNM tool failed to identify nearly two-thirds of sustained severe acute maternal morbidity cases and more than one-third of maternal deaths. Disease-based criteria remain important, and using only organ dysfunctionbased criteria would lead to underestimating severe acute maternal morbidity.
\end{abstract}

Abbreviations: MNM, Maternal Near Miss; SAMM, severe acute maternal morbidity; WHO, World Health Organization.

\section{Introduction}

Prevention of maternal deaths is one of the major goals in global maternity care $(1,2)$. Maternal mortality is used as a quality marker for obstetric care $(3,4)$. Fortunately, maternal deaths have become rare events in high-income countries $(5,6)$. Therefore, other markers including severe acute maternal morbidity (SAMM) have been introduced

\section{Key Message}

In the Netherlands, the organ dysfunction-based criteria of the WHO Maternal Near Miss Tool failed to identify nearly two-thirds of sustained severe acute maternal morbidity cases and more than one-third of maternal deaths. 
to monitor the quality of obstetric care (5). SAMM is a stage in the continuum between complication and mortality, occurs more frequently than mortality $(3,7)$ and may have similar associated factors (1).

To arrive at a universal and discriminatory definition of severe maternal morbidity, the World Health Organization (WHO) proposes the term "Maternal Near Miss" (MNM). MNM is defined as a woman who nearly died but survived a complication that occurred during pregnancy, childbirth or within 42 days of termination of pregnancy (8). With the aim of creating uniform criteria to detect and monitor MNM and enable cross-country comparisons (1), WHO developed the Maternal Near Miss Tool (MNM Tool) (Figure 1) (8). This tool summarizes three main types of criteria to identify MNM: five disease-based criteria (A0-4, "A-criteria"); four critical interventions (B0-3, "B-criteria") and seven organ dysfunc- tion criteria (C0-6, "C-criteria") $(1,8)$. According to WHO, $\mathrm{C}$-criteria are the most promising markers to detect MNM, since organ dysfunction is the ultimate step in the continuum from complication to death $(1,9,10)$. WHO claims that C-criteria are sensitive enough to pick up severe (lifethreatening) cases and specific enough not to include "unnecessary," less severe (potentially life-threatening) complications, so as to arrive at a manageable workload for audit purposes.

The MNM tool applicability was previously studied in single institutions in a variety of settings, including Brazil, Malawi and Tanzania (10-12). These studies indicate that the MNM tool in general and the organ dysfunction criteria in particular detect only a small proportion of all severe morbidity (Brazil 12\%, Malawi 22\%, Tanzania $42 \%$ ). In addition, the largest assessment of the MNM tool to date, performed by WHO, did not include any

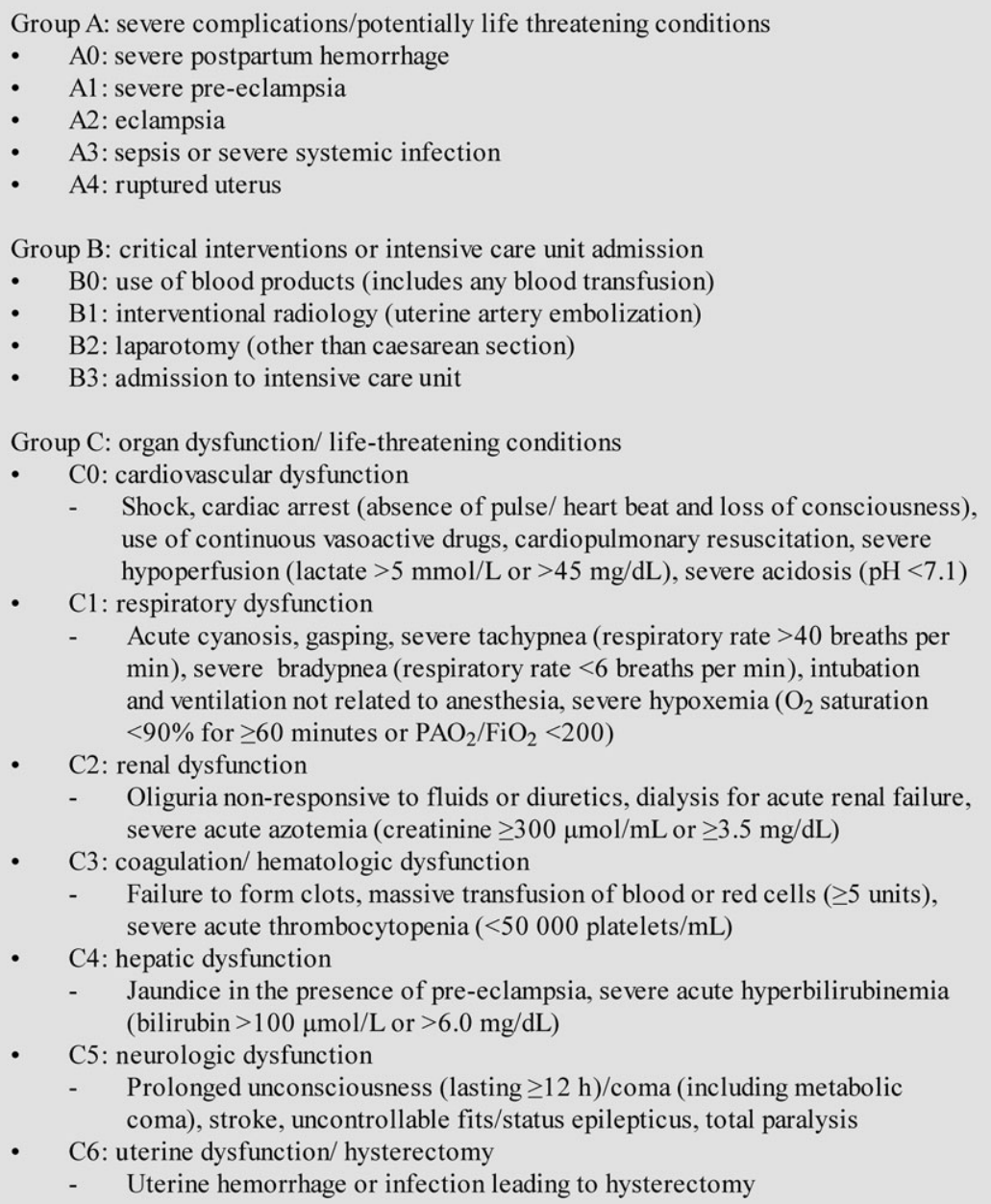

Figure 1. Maternal Near Miss tool groups and subcategories (8). 
high-income European country (13). This means that the applicability of the MNM tool in these countries is currently not known.

Therefore, this study was performed to validate the MNM tool in the Netherlands, as an example of a highincome European country. Our aims were to investigate the applicability of the MNM tool and to determine whether organ dysfunction criteria are suitable as markers to identify severe morbidity.

\section{Material and methods}

We applied the WHO MNM tool to a previously collected cohort of women who sustained severe morbidity in the Netherlands (LEMMoN study) (14). Data collection was done prospectively between 1 August 2004 and 1 August 2006. All 98 hospitals with a maternity unit participated: 10 tertiary care centers, 33 non-university teaching hospitals and 55 general hospitals. Inclusion criteria were: intensive care unit admission, uterine rupture, eclampsia or HELLP (hemolysis, elevated liver enzymes, low platelets) syndrome with liver hematoma or rupture, major obstetric hemorrhage (defined as a need for four and more units of blood for transfusion) and a miscellaneous group of cases of severe morbidity in the opinion of the treating obstetrician, which could not be included in groups 1-4. Maternal deaths during the study period were also included. More detailed information about data collection of the LEMMoN study was described previously (14).

The MNM groups and subcategories are shown in Figure 1. The MNM tool was applied to each LEMMoN case. Fourteen $(0.6 \%)$ cases were excluded due to insufficient data for application of the tool. Each case could be part of more than one MNM group and fulfill several subcategories, which are called events. For example, a woman with major postpartum hemorrhage who received six units of packed cells, fulfilled disease-based (event: severe postpartum hemorrhage), intervention-based (event: use of blood products) and organ dysfunction-based (event: coagulation dysfunction defined as $\geq 5$ units of blood) criteria. Based on WHO terminology, disease-based criteria are used to identify potentially life-threatening conditions, whereas organ dysfunction-based criteria identify life-threatening conditions.

For each woman, the following parameters were available: maternal age, parity, body mass index, smoking habits during pregnancy, socio-economic status indicator according to postal code (14), ethnic origin as defined by Statistics Netherlands (15), mode of delivery, quantity of blood loss and number of received blood products. If additional information was necessary, anonymized patient records were also available. Two investigators (IK, HB) independently applied the MNM tool to all SAMM cases. Afterwards, their results were compared. Differences in interpretation were discussed with the entire research team until consensus about categorization was reached. Cases with incomplete or missing information were discussed within the research team to prevent misclassification or unnecessary exclusion.

Primary outcomes were the number of women and events detected within the three main MNM groups and subcategories (A0-4, B0-3, C0-6). Based on these and the numbers of maternal deaths, case-fatality rates were calculated. A comparison was made between women with potentially life-threatening conditions and those with lifethreatening conditions.

In this study we used anonymous data from the LEMMoN study that cannot be related to any individual. The LEMMoN study was approved by the medical ethics committee of the Leiden University Medical Center (P04-020; 8 March 2004) (14).

\section{Statistical analysis}

The numeric parameters were compared using independent sample $t$-tests. Statistical analysis was performed using SPSS statistics, version 20.0 (IBM Corp., Armonk, NY, USA).

\section{Results}

In the period of the LEMMoN study, there were 371623 deliveries in the Netherlands (15). A total of 2552 SAMM cases were reported $(0.7 \%$ of all deliveries). General characteristics of the 2538 women assessed by the MNM tool are shown in Table 1. Of these, 2308 women (90.9\%) fulfilled one or more disease based-criteria, 2116 (83.4\%) one or more intervention based-criteria and 1024 (40.3\%) one or more organ dysfunction-based criteria. In total there were 7007 events reported, of which 2638 (37.6\%) were disease-based, 3190 (45.5\%) intervention-based and 1179 (16.8\%) organ dysfunction-based. Table 2 shows the number of events in each subcategory.

During the study period, 48 deaths occurred. Of these, $23(47.9 \%)$ fulfilled disease-based, 33 (68.8\%) intervention-based and $31(64.6 \%)$ organ dysfunctionbased criteria. There were five maternal deaths (10.4\%) that could not be classified into any MNM group: suicide, acute asthma exacerbation, pancreas carcinoma, liver cirrhosis and massive pulmonary embolism, respectively. The case-fatality rate was 23/2308 (1.0\%) for cases that only fulfilled disease-based criteria, 33/2116 (1.6\%) for cases that additionally fitted intervention-based criteria and $31 / 1024(3.0 \%)$ for cases fulfilling organ dysfunction criteria. 
Table 1. Characteristics of women in the study.

\begin{tabular}{|c|c|c|}
\hline & $n$ & $\%$ \\
\hline \multicolumn{3}{|l|}{ Age (years) } \\
\hline$<20$ & 31 & 1.2 \\
\hline $20-34$ & 1770 & 69.8 \\
\hline $35-39$ & 589 & 23.2 \\
\hline$\geq 40$ & 122 & 4.8 \\
\hline Unknown & 26 & 1.0 \\
\hline \multicolumn{3}{|l|}{ Parity } \\
\hline 0 & 1259 & 49.6 \\
\hline 1 & 867 & 34.2 \\
\hline$\geq 2$ & 390 & 16.3 \\
\hline Unknown & 22 & 0.9 \\
\hline \multicolumn{3}{|l|}{ Mode of delivery } \\
\hline Induction of labor & 1196 & 47.1 \\
\hline Spontaneous & 1118 & 44.1 \\
\hline Cesarean section & 1058 & 41.7 \\
\hline Ventouse/forceps & 300 & 11.8 \\
\hline Breech delivery & 10 & 0.4 \\
\hline Unknown & 11 & 0.4 \\
\hline \multicolumn{3}{|l|}{ Quantity of blood loss (L) } \\
\hline$<1$ & 688 & 27.1 \\
\hline $1.0-4.9$ & 1390 & 54.8 \\
\hline $5.0-9.9$ & 159 & 6.3 \\
\hline$\geq 10$ & 31 & 1.2 \\
\hline Unknown & 271 & 10.7 \\
\hline \multicolumn{3}{|l|}{ Smoking during pregnancy } \\
\hline Yes & 176 & 6.9 \\
\hline No & 1294 & 51.0 \\
\hline Unknown & 1068 & 42.1 \\
\hline \multicolumn{3}{|l|}{ Body mass index } \\
\hline$<18.5$ & 60 & 2.4 \\
\hline $18.5-24.9$ & 969 & 38.2 \\
\hline $25.0-29.9$ & 386 & 15.2 \\
\hline$\geq 30$ & 238 & 8.6 \\
\hline Unknown & 905 & 35.6 \\
\hline \multicolumn{3}{|c|}{ Socio-economic status indicator } \\
\hline Low & 701 & 27.6 \\
\hline Middle & 991 & 39.1 \\
\hline High & 520 & 20.5 \\
\hline Unknown & 326 & 12.8 \\
\hline \multicolumn{3}{|l|}{ Received packed cells $(n)$} \\
\hline 0 & 734 & 28.9 \\
\hline$<5$ & 946 & 38.4 \\
\hline $5-9$ & 542 & 37.3 \\
\hline 10-19 & 189 & 7.4 \\
\hline$\geq 20$ & 50 & 2.0 \\
\hline Unknown & 77 & 3.0 \\
\hline \multicolumn{3}{|l|}{ Ethnic origin } \\
\hline Netherlands & 1862 & 73.4 \\
\hline Morocco & 116 & 4.6 \\
\hline Surinam/Dutch Antilles & 111 & 4.3 \\
\hline Sub-Sahara Africa & 93 & 3.7 \\
\hline Turkey & 87 & 3.4 \\
\hline Indonesia & 112 & 4.4 \\
\hline South-America & 10 & 0.4 \\
\hline Other Western ${ }^{a}$ & 113 & 4.5 \\
\hline Unknown & 34 & 1.3 \\
\hline
\end{tabular}

aJapan, USA, Canada.
Comparison between potentially life-threatening (women fulfilling disease-based criteria) and life-threatening (women fulfilling organ dysfunction criteria) conditions is shown in Table 3. In the life-threatening group the following parameters were significantly different: higher maternal age, longer duration of hospital stay, lower body mass index, lower maximum diastolic blood pressure, more blood loss and transfusion of packed cells.

\section{Discussion}

To our knowledge, this is the first study evaluating the application of the WHO MNM tool in a high-income European country. Our findings show that the organ dysfunction criteria failed to identify nearly $60 \%$ of severe maternal morbidity cases. In contrast, disease-based criteria detected more than $90 \%$ of the SAMM cases.

Our results are comparable to other studies from different settings. A cross-sectional study in Brazil found that only 10 of $84(12 \%)$ MNM cases fulfilled organ dysfunction criteria. Two more recent studies (2013) in Malawi and Tanzania found organ dysfunction detection percentages of 22\% (84 of 386 women) and 42\% (103 of 248 women). Importantly, case-fatality rates for the study populations in these countries were $3.2 \%$ (Brazil), 12\% (Malawi) and 13\% (Tanzania), which indicates that it is justified to state that all women with SAMM in these countries actually have "life-threatening" conditions. The low detection results were attributed to absence of sophisticated laboratory diagnostics and lack of manpower to perform extensive clinical monitoring in low-income countries $(10,12)$. In the Netherlands, however, such laboratory diagnostics and human resources are available. Therefore, our nationwide results indicate that organ dysfunction-based criteria also underperform in a setting with sufficient resources.

The differentiation between "potentially life-threatening" and "life-threatening" conditions shows a significantly higher maternal age, longer duration of hospital stay, more units of packed cells, more blood loss and higher parity in the life-threatening group. These determinants are known factors associated with maternal morbidity or mortality $(12,16)$. Mean body mass index and maximum diastolic blood pressure were higher in the potentially life-threatening group, which may be explained by the fact that obesity is a risk factor for high blood pressure and (pre)eclampsia $(16,17)$.

The case-fatality rate was highest for organ dysfunction criteria (potentially life-threatening $1.0 \%$, life-threatening $3.0 \%$ ), which suggests that the WHO terminology "lifethreatening" may be justified. However, attributing this terminology to this relatively limited difference in casefatality rate can be considered highly arbitrary, since both 
Table 2. Overview after application of the Maternal Near Miss Tool.

\begin{tabular}{|c|c|c|c|c|}
\hline Category & SAMM cases $(\%)$ & Events (\%) & Subcategory & Events (\%) \\
\hline \multirow[t]{5}{*}{ A: disease } & $2308(90.9)$ & $2638(37.6)$ & $0: \mathrm{PPH}$ & $1635(61.9)$ \\
\hline & & & 1: Pre-eclampsia & $414(15.7)$ \\
\hline & & & 2: Eclampsia & $242(9.2)$ \\
\hline & & & 3: Infection/sepsis & $118(4.5)$ \\
\hline & & & 4: Ruptured uterus & $229(8.7)$ \\
\hline \multirow[t]{4}{*}{ B: intervention } & $2116(83.4)$ & $3190(45.5)$ & 0 : Any blood products & $1738(57.5)$ \\
\hline & & & 1: Interventional radiology & $111(3.7)$ \\
\hline & & & 2: Laparotomy & $267(8.8)$ \\
\hline & & & 3: Admission to ICU & $909(30.0)$ \\
\hline \multirow[t]{7}{*}{ C: organ failure } & $1024(40.3)$ & $1179(16.8)$ & 0: Cardiovascular & $165(12.5)$ \\
\hline & & & 1: Respiratory insufficiency & $115(8.7)$ \\
\hline & & & 2: Renal & $26(2.0)$ \\
\hline & & & 3: Coagulation/Hematologic & $846(63.8)$ \\
\hline & & & 4: Hepatic insufficiency & $27(2.0)$ \\
\hline & & & 5: Neurologic & $33(2.5)$ \\
\hline & & & 6: Hysterectomy & $113(8.5)$ \\
\hline Total & & 7007 & & \\
\hline
\end{tabular}

$\mathrm{ICU}$, intensive care unit; PPH, postpartum hemorrhage; SAMM, severe acute maternal morbidity.

Table 3. Comparison of potentially life-threatening and lifethreatening groups.

\begin{tabular}{lccc}
\hline & $\begin{array}{l}\text { Life- } \\
\text { threatening }\end{array}$ & $\begin{array}{l}\text { Potentially } \\
\text { life-threatening }\end{array}$ & p-value \\
\hline Maternal age (years) & $31.9(5.0)$ & $31.5(4.9)$ & 0.043 \\
Duration of hospital & $10.4(12.0)$ & $7.4(6.9)$ & 0.000 \\
$\quad$ stay (days) & & & \\
BMl & $24.4(5.2)$ & $25.1(5.5)$ & 0.017 \\
Parity & $2.9(11.1)$ & $2.2(7.2)$ & 0.070 \\
Maximum DBP (mmHg) & $85.4(16.9)$ & $89.8(19.0)$ & 0.000 \\
Blood loss (mL) & $3415(2715)$ & $1639(1151)$ & 0.000 \\
Units of packed cells $(n)$ & $7.5(6.4)$ & $2.4(2.0)$ & 0.000 \\
Birth weight infant $(g)$ & $3034(1031)$ & $3069(1051)$ & 0.434 \\
\hline
\end{tabular}

$\mathrm{BMI}$, body mass index; DBP, diastolic blood pressure.

(or neither) of these case-fatality rates could be interpreted as "life-threatening". We also found that the MNM tool failed to detect $35 \%$ of all maternal deaths. This clearly shows that these criteria are not able to detect every life-threatening condition.

Finally, further analysis of women in each organ dysfunction subgroup (specifically C3; coagulation/hematologic dysfunction criteria, see Figure 1) indicates that $76 \%$ of all included women in the organ dysfunction group (781/1024) would have been included on the basis of a single criterion: massive transfusion of $\geq 5$ units packed cells. This means that $75 \%$ of all women included on the basis of the organ dysfunction criteria would have been detected with one relatively simple criterion. This underlines the extreme importance of well-organized blood transfusion guidelines and services (18).
One limitation of our study is that we used data from a previous study, designed to detect SAMM according to different criteria and not to assess the MNM tool. An expert panel of obstetricians and the national Maternal Mortality Committee of the Dutch Society of Obstetrics and Gynecology defined the LEMMoN study inclusion criteria, based on previous international studies. Considering that the MNM tool missed 60\% of these differently defined SAMM cases, the consequence can only be a relative overreporting of MNM within the LEMMoN study. This means that it is unlikely that cases that could have been detected by the MNM tool would have been missing from this nationwide study.

A second limitation was incomplete or missing information. Therefore, cases with incomplete information were discussed and assessed by our research group to prevent bias. As a consequence, the number of excluded cases $(0.6 \%)$ could also be minimized. We believe that the large number of cases in this reliable dataset provides a unique and solid base for validating the MNM tool in a high-income country. A third limitation is the relatively old dataset, but we have no reason to consider that our findings would be different in a cohort of more recent date.

Although the intervention-based criteria were able to identify a considerable number of SAMM and mortality cases, these criteria are not suitable for international comparison studies because of different criteria for transfusing blood products and indications to perform laparotomy. Admission into intensive care units and interventional radiology, such as embolization of the uterine arteries, are not present in all settings, and - where present - access 
depends on local protocols. Our advice would be to further refine the potentially life-threatening criteria of the WHO MNM tool, since these make early medical intervention possible with the intention of preventing lifethreatening conditions and averting maternal deaths.

In the Netherlands, where advanced laboratory and clinical monitoring are available, the organ dysfunctionbased criteria of the WHO MNM tool fail to identify nearly two-thirds of SAMM cases and more than onethird of maternal deaths. Disease-based criteria remain important, and using only organ dysfunction-based criteria to detect MNM cases would lead to an underestimation of severe maternal morbidity. Therefore, we propose focusing future discussions on potentially life-threatening conditions in the MNM tool in order to establish universal disease-based criteria to prevent life-threatening maternal morbidity.

\section{Acknowledgments}

The authors wish to thank the 98 local hospital coordinators for kindly participating in the LEMMoN study, reporting cases on a monthly basis, and supervising the data collection process.

\section{Funding}

The authors hereby state that no external funding was used for this study.

\section{References}

1. Say L, Souza JP, Pattinson RC. Maternal near miss towards a standard tool for monitoring quality of maternal health care. Best Pract Res Clin Obstet Gynaecol. 2009;23:287-96.

2. Amaral E, Souza JP, Surita F, Luz AG, Sousa MH, Cecatti JG, et al. A population-based surveillance study on severe acute maternal morbidity (near-miss) and adverse perinatal outcomes in Campinas, Brazil: the Vigimoma Project. BMC Pregnancy Childbirth. 2011;11:9.

3. Tuncalp O, Hindin MJ, Souza JP, Chou D, Say L. The prevalence of maternal near miss: a systematic review. BJOG. 2012;119:653-61.

4. Ps R, Verma S, Rai L, Kumar P, Pai MV, Shetty J. "Near miss" obstetric events and maternal deaths in a tertiary care hospital: an audit. J Pregnancy. 2013;2013:393758.

5. Pattinson RC, Hall M. Near misses: a useful adjunct to maternal death enquiries. Br Med Bull. 2003;67:231-43.
6. Jayaratnam S, De CC, Howat P. Developing an assessment tool for maternal morbidity "near-miss" - a prospective study in a large Australian regional hospital. Aust N Z J Obstet Gynaecol. 2011;51:421-5.

7. Mantel GD, Buchmann E, Rees H, Pattinson RC. Severe acute maternal morbidity: a pilot study of a definition for a near-miss. Br J Obstet Gynaecol. 1998;105:985-90.

8. World Health Organization. Evaluating the quality of care for severe pregnancy complications. The WHO near-miss approach for maternal health. Geneva: WHO, 2011.

9. Say L, Pattinson RC, Gulmezoglu AM. WHO systematic review of maternal morbidity and mortality: the prevalence of severe acute maternal morbidity (near miss). Reprod Health. 2004;1:3.

10. Morse ML, Fonseca SC, Gottgtroy CL, Waldmann CS, Gueller E. Severe maternal morbidity and near misses in a regional reference hospital. Rev Bras Epidemiol. 2011;14:310-22.

11. van den Akker T, Beltman J, Leyten J, Mwagomba B, Meguid T, Stekelenburg J, et al. The WHO maternal near miss approach: consequences at Malawian District level. PLoS ONE. 2013;8:e54805.

12. Nelissen E, Mduma E, Broerse J, Ersdal H, Evjen-Olsen B, van Roosmalen J, et al. Applicability of the WHO maternal near miss criteria in a low-resource setting. PLoS ONE. 2013;8:e61248.

13. Souza JP, Gulmezoglu AM, Vogel J, Carroli G, Lumbiganon P, Qureshi Z, et al. Moving beyond essential interventions for reduction of maternal mortality (the WHO Multicountry Survey on Maternal and Newborn Health): a cross-sectional study. Lancet. 2013;381:1747-55.

14. Zwart JJ, Richters JM, Ory F, de Vries JI, Bloemenkamp KW, van Roosmalen J. Severe maternal morbidity during pregnancy, delivery and puerperium in the Netherlands: a nationwide population-based study of 371,000 pregnancies. BJOG. 2008;115:842-50.

15. Statistics Netherlands (CBS). Statline, Central Bureau of Statistics, 2013. Available online at: http://statline.cbs.nl/ Statweb/?LA=en (accessed 4 January 2013).

16. Witteveen T, Zwart JJ, Gast KB, Bloemenkamp KW, van Roosmalen J. Overweight and severe acute maternal morbidity in a low-risk pregnant population in the Netherlands. PLoS ONE. 2013;8:e74494.

17. Robinson HE, O’Connell CM, Joseph KS, McLeod NL. Maternal outcomes in pregnancies complicated by obesity. Obstet Gynecol. 2005;106:1357-64.

18. Hendriks J, Zwart JJ, Briët E, Brand A, van Roosmalen J. The clinical benefit of blood transfusion; a hypothetical case-control study based on a nationwide survey of severe maternal morbidity. Vox Sang. 2013;104:234-9. 